\title{
BMJ Open Effectiveness of self-testing kits availability on improving HIV testing frequency for chinese men who have sex with men and their sexual partners: a protocol for a multicenter randomised controlled trial
}

\author{
Ci Zhang, ${ }^{1}$ Xianhong Li, ${ }^{1}$ Deborah Koniak-Griffin, ${ }^{2}$ Lloyd A. Goldsamt, ${ }^{3}$ Jing Zhou ${ }^{1}$
}

To cite: Zhang C, Li X, KoniakGriffin D, et al. Effectiveness of self-testing kits availability on improving HIV testing frequency for chinese men who have sex with men and their sexual partners: a protocol for a multicenter randomised controlled trial. BMJ Open 2018;8:e024423. doi:10.1136/ bmjopen-2018-024423

- Prepublication history for this paper is available online. To view these files, please visit the journal online (http://dx.doi org/10.1136/bmjopen-2018024423).

Received 26 May 2018 Revised 29 August 2018 Accepted 12 October 2018

Check for updates

(c) Author(s) (or their employer(s)) 2018. Re-use permitted under CC BY-NC. No commercial re-use. See rights and permissions. Published by BMJ.

${ }^{1}$ Xiangya Nursing School of Central South University, Changsha, China

${ }^{2}$ School of Nursing of University of California at Los Angeles, Los Angeles, California, USA

${ }^{3}$ New York University Rory Meyers College of Nursing, New York, New York, USA

Correspondence to Dr Xianhong Li; xianhong_li228@hotmail.com

\section{ABSTRACT}

Introduction HIV epidemic is increasing among men who have sex with men (MSM) in China, yet HIV testing uptake remains low. As an emerging approach, HIV self-testing (HIVST) has the potential to promote HIV testing coverage and frequency in this population. However, evidence of the effectiveness on implementation of HIVST among Chinese MSM and their sexual partners is scarce.

Methods and analysis The randomised controlled trial will be performed in Changsha, Changde, Shaoyang and Yiyang, Hunan province, China, recruiting 184 recent testers (men who had at least one HIV test within the past 2 years) and 26 non-recent testers (men who did not have HIV tests within 2 years or never had an HIV test). Eligible men will be randomly divided 1:1 into two groups: intervention (with free HIVST kits plus site-based HIV testing services) and control (site-based HIV testing services only). Participants in the intervention group will be provided with two free finger-prick-based HIVST kits, and can apply for two to four kits every 3 months for 1 year. Participants in both groups will complete questionnaires via WeChat at five separate times: baseline, third, sixth, ninth and twelfth month. The primary outcome is the mean number of HIV tests for MSM over the 12-month study period. The secondary outcome is the mean number of HIV tests for sexual partners of MSM over the 12-month study period. The tertiary outcomes are the self-reported proportion of consistent condom usage for anal sex, and the numbers of sexual partners during the 12-month study period.

Ethics and dissemination The study has been approved by the Institutional Review Board of Behavioural and Nursing Research in Xiangya School of Nursing of Central South University, China (2018002). Study results will be disseminated through conferences and academic journals. Trial registration number ChiCTR1800015584; Preresults.

\section{INTRODUCTION}

Globally, HIV remains a major public health threat, with a total of approximately
Strengths and limitations of this study

- No study has reported effectiveness of free HIV self-testing (HIVST) kits availability on increasing HIV testing frequency among men who have sex with men (MSM) in China.

- One major innovation of this study is to determine effectiveness of distribution of HIVST kits from MSM to their sexual partners, which will provide evidence to guide the application of HIVST to promote partner testing and improve HIV testing coverage.

- Findings from this study will have potential to help policymakers to scale up the HIVST approach to reach more high-risk individuals and could be used to develop contextualised HIV prevention strategies for Chinese MSM.

- In this longitudinal study, maintaining MSM for 12 months is difficult and thus loss to follow-up is possible.

- Data about sexual partners' use of HIVST kits which will be obtained indirectly by report of MSM may be inclined to bias.

36.7 million individuals infected at the end of 2015. ${ }^{1}$ Despite great efforts to improve HIV prevention and treatment, the annual number of newly HIV-infected individuals remains around 2.2 million over the past 5 years. $^{2}$ Notably, the transmission rate of HIV among men who have sex with men (MSM) is alarmingly high globally, accounting for $30 \%$ of all infected individuals. ${ }^{3}$ China has also faced a severe HIV epidemic among MSM, ${ }^{4}$ with the HIV prevalence among MSM increasing nearly nine times from $0.9 \%$ in 2003 to $8.0 \%$ in $2015,{ }^{56}$ and $25.5 \%$ of new infections were attributed to MSM in $2017^{7}$

In 2014, the Joint United Nations Programme on HIV/AIDS (UNAIDS) set up the '90-90-90' goal aiming to control the HIV 
epidemic by 2020 , with $90 \%$ of HIV-infected individuals getting tested and diagnosed, $90 \%$ of diagnosed individuals receiving treatment and $90 \%$ of individuals under treatment achieving viral suppression. ${ }^{8}$ However, only $60 \%$ of HIV-positive individuals have been tested around the world, ${ }^{3}$ far from reaching the first goal. The situation in China is similar. A recent meta-analysis showed that $62 \%$ of MSM had not taken an HIV test over the past 12 months, and nearly $50 \%$ had never been tested in their lifetime. ${ }^{9}$ Lack of adequate HIV testing services, multiple sexual partners and frequent high-risk sexual behaviours have exacerbated HIV prevalence among MSM and may lead to further transmission to the general population via sexual contacts with female sexual partners. ${ }^{10}$ Moreover, delayed HIV testing and delayed antiretroviral treatment may increase the probability of HIV/AIDS-related deaths. ${ }^{12}$ Thus, it is essential to promote HIV testing, especially among MSM.

As a complementary approach, HIV self-testing (HIVST) has significant potential to scale up HIV testing coverage. It can be used by individuals to collect specimens (their own blood or saliva), perform a test and read the test results by themselves. ${ }^{13}$ With the characteristics of convenience, privacy and confidentiality, ${ }^{14-16}$ HIVST may be very attractive to MSM who are impacted by HIV/ AIDS-related stigma and discrimination; limited transportation; and/or reluctance to wait when taking the test at the traditional sites, such as the Centre for Disease Prevention and Control (CDC), sites of voluntary counselling and testing services for AIDS and hospitals. ${ }^{17} \mathrm{~A}$ recent review indicated that HIVST kits had high acceptability $(>67 \%)$ among MSM of the 14 included studies ${ }^{18}$ not specific to oral fluid based or blood based. However, the usage rate of HIVST kits among MSM was low in certain countries, with reported rates of $3.5 \%$ in France, $6.1 \%$ in China and $11.6 \%$ in the USA. ${ }^{19-21}$ The barriers to use included worry about a positive result, the relatively high cost of kits and concerns about correct usage of the kits. ${ }^{22}$

Data released by UNAIDS (2017) underlines that reaching MSM and their partners with HIV testing services may have the potential to control the HIV epidemic. ${ }^{23}$ In the USA, a randomised controlled trial (RCT) showed that the availability of HIVST could increase HIV testing uptake among MSM. ${ }^{24}$ A previous RCT in Australia also indicated that HIVST could increase the HIV testing frequency among MSM, especially in delayed testers (those who had never received HIV testing or had not been tested within 2 years).$^{25}$ Moreover, a previous US study showed that $79 \%-91 \%$ of MSM were willing to distribute HIVST kits to their sexual partners. ${ }^{26}$ Serostatus disclosure to their regular or casual sexual partners after testing with HIVST kits would promote safe sexual practices if the testing results were positive. ${ }^{27} 28$ Thus, distribution of HIVST kits from MSM to their sexual partners could be an effective way to expand HIV testing and reduce highrisk sexual behaviours among MSM. ${ }^{27} 28$

In accordance with this strategy, a guideline on HIVST was issued by the WHO in 2016 to promote HIV testing and partner disclosure. ${ }^{13}$ However, in the guideline, there was only evidence from two RCTs to support the effectiveness of HIVST kits for improving HIV testing frequency among MSM. ${ }^{24}{ }^{25}$ Further research is urgently needed to determine the effects of providing free HIVST kits to MSM and their sexual partners in China.

\section{METHODS}

\section{Study objectives}

This RCT is designed to determine whether the distribution of HIVST in an intervention group compared with a control group improves the frequency of HIV testing among MSM (primary aim) and their sexual partners (male and female) (secondary aim). The study also aims to explore whether HIVST reduces high-risk sexual behaviours of MSM (tertiary aim). Outcomes will be compared between participants in the intervention and control groups who have histories of recent and non-recent HIV testing.

\section{Study settings}

This study will be conducted in four cities within Hunan Province, namely Changsha (the capital of the province), Changde, Shaoyang and Yiyang. The settings include urban and rural areas. Delivery services of free HIVST kits are unavailable through any organisations in these cities. HIV prevalence in Hunan Province ranks ninth nationally in mainland China. ${ }^{29}$ The number of patients with HIV/ AIDS in Hunan Province was about 24000 in 2016, with 4974 newly infected.$^{30}$ Notably, $64.8 \%$ of the new patients were infected through male-to-male sexual intercourse. ${ }^{30}$ In our unpublished cross-sectional study, 37.7\% of MSM had never tested for HIV in Hunan Province.

\section{Participants}

Two subgroups of MSM will be recruited: recent testers (men who had at least one HIV test within the last 2 years) and non-recent testers (men who did not have HIV tests within the last 2 years or never had an HIV test). Zuo An Cai Hong, a gay-friendly community-based organisation $(\mathrm{CBO})$, with seven sublocations in the participating settings of Hunan Province, will collaborate in this study. The CBO provides rapid HIV tests, counselling and referral for MSM, and it conducts around 1500-2000 HIV tests each year. Thus, the feasibility of the study recruitment is supported.

\section{Inclusion criteria}

Individuals are eligible for participation if they meet the following criteria: (1) born as a male; (2) 18 years of age or older; (3) reporting condomless anal or oral sex with men in the past 3 months; (4) HIV negative by rapid HIV testing at screening; (5) planning to reside in Hunan Province during the next year; (6) possessing a smartphone and adept in using WeChat; and (7) voluntarily agreeing to participate in the study and provide written informed consent. 


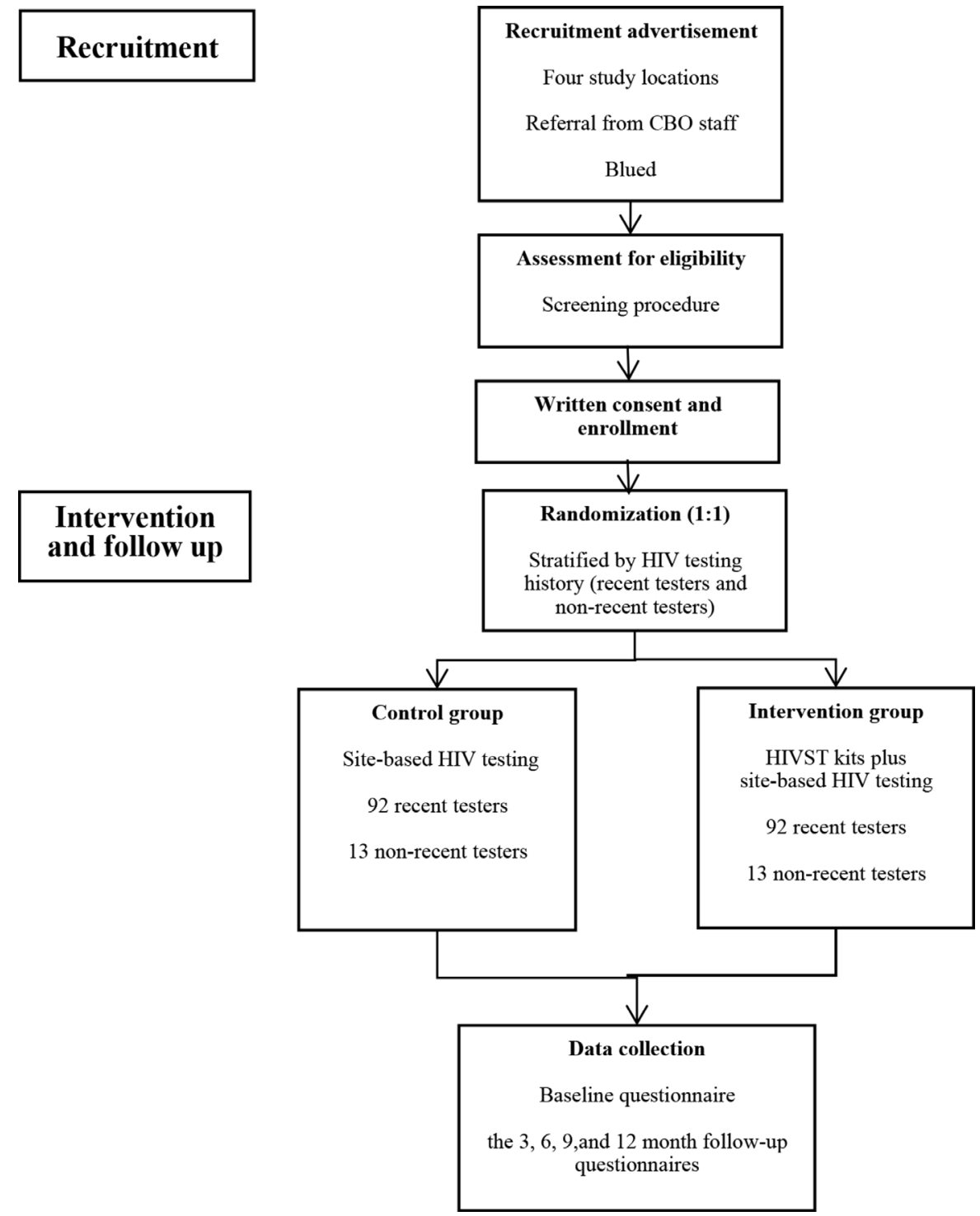

Figure 1 Flow of recruitment and intervention. CBO, community-based organisation; HIVST, HIV self-testing.

\section{Exclusion criteria}

Individuals will be excluded if they: (1) are participating in other research programmes; (2) cannot speak and/or read Chinese; or (3) score higher than 35 on the Brief Psychiatric Rating Scale. ${ }^{31}$

\section{Study procedure}

Study design

A multisite RCT design will be used to determine the effectiveness of HIVST on improving HIV testing frequency among Chinese MSM and their sexual partners, and to explore whether HIVST reduces high-risk sexual behaviours of MSM in China. Participants will be randomly assigned to intervention and control groups, with a 1:1 ratio (by CZ). The intervention group will be provided with free HIVST kits and site-based HIV testing services for 1 year, and participants in the control group will have access to site-based HIV testing for 1 year (the control group will also receive the HIVST kits after the study concludes) (figure 1).

\section{Recruitment}

Participants will be recruited starting from December 2017 through posting of flyers (1) in the office and waiting areas of the four study locations, and (2) on Blued (a social media site for MSM), the instant online-chatting platform of QQ and WeChat (public number of Zuo An Cai Hong). CBO staff also will provide study information to MSM who are coming for testing or counselling. A WeChat account and a phone number will be established solely for study online consultation and follow-up.

MSM who express interest in this study will be contacted by the research assistant (RA) (CZ) via WeChat or cellphone for purposes of scheduling an appointment at the nearby sublocation of the $\mathrm{CBO}$ to complete the screening procedure and receive a rapid HIV test following oral consents. Figure 1 presents the recruitment flow chart.

\section{Randomisation}

Securing of written informed consent will be obtained from eligible MSM prior to randomisation into the 
intervention group and control group. Computer-generated randomised number tables developed by SPSS V.18.0 (by JZ) will be used for this purpose. Two separate randomised number tables will be used for recent testers and non-recent testers.

\section{Blinding}

During the screening and informed consent procedure, the RAs will be blinded to the randomisation number tables, which will be sealed in opaque and sequentially numbered envelopes. After screening procedure and written informed consent, the envelope will be opened and the group assignment will be exposed to both the participants and the RAs. Throughout the project, the data analysts will be blinded to code numbers representing the group assignments.

\section{Intervention group}

Participants in the intervention group will have access to free HIVST kits in addition to site-based HIV testing services. They will be encouraged to perform HIV tests for themselves and their sexual partners using the HIVST kits. At enrolment, participants will be provided two HIVST kits along with detailed instructions and information without any face-to-face training from our study. Instructions and additional information will also be posted in the official account of Zuo An Cai Hong on WeChat (https://mp.weixin.qq.com/s/vi9Nl-uLOeWnoJajvsq63Q). Participants in the intervention group will be asked to submit a photo of each test result, following which they can apply to receive new kits. A cross-sectional study in China indicated that the mean number of sexual partners of MSM was 4.79 during the past 6 months. ${ }^{32}$ Given the repeated tests, distribution to sexual partners and the recommendation of 2-4 tests per year for highrisk MSM, ${ }^{33}{ }^{34}$ participants can apply for $2-4$ kits every 3 months, and a maximum of 12 kits for 1 year. For participants' convenience, these kits can be obtained at study sites or through express delivery. With 24 hours' notice, the kits will be delivered in private packages without any information about HIV/AIDS or testing visible on the packaging. To encourage MSM to send back the photos of their results, participants will be charged a $30 ¥$ (the equivalent of US\$5) deposit for each kit, which will be returned immediately after the RA receives the result photos. Each kit will be numbered sequentially on the outer packing before distribution for monitoring purposes. In addition, MSM can also purchase HIVST kits online if they need more than four kits every 3 months.

\section{The product of self-testing kits}

Selection of the type of HIVST kits (oral fluids from mouth swabs vs blood samples from finger pricking) was made with consideration of research findings. Previous studies indicate that $5 \%-10 \%$ of participants made procedural errors, ${ }^{35} 36$ and $10 \%$ requested additional help beyond the provided demonstration and instructions when using oral self-testing kits. ${ }^{36}$ Thus, a finger-prick-based
HIVST kit, a third generation of Alere Determine HIV 1/2 rapid assay (Alere Medical Co., Japan), approved by the US Food and Drug Administration and China's State Food and Drug Administration, was selected for use in this study. ${ }^{37} 38$ This test can detect HIV $1 / 2$ antibodies in blood specimens at 6 weeks postinfection. ${ }^{3}$ The specimen is obtained by pricking a finger using a disposable peripheral blood needle made by STERiLANCE in China. Blood is collected by EDTA capillary tube and dropped on a specimen reaction zone of the HIVST kit, and then Alere Determine Chase solution is added in the reaction zone. ${ }^{39}$ The result can be read in $15-60$ min. ${ }^{39}$ The specificity and sensitivity of the Alere Determine HIV $1 / 2$ are $99.68 \%$ and $100 \%$, respectively. ${ }^{40}$ Every kit is accompanied by instructions including testing procedure, interpretation of results and notes. The HIVST is preliminary for screening, and a positive result requires confirmation by enzyme-linked immunoassay in the CDC laboratory.

\section{Support hotlines}

Participants will be able to contact RAs by WeChat or a 24-hour telephone support line to obtain consultation on the HIVST administration and interpretation of results. Records will be maintained on the purpose of the consultation.

\section{Control group}

Participants in the control group will receive the usual care provided at the CDC, CBO-based rapid HIV testing sites or local hospitals where they may receive HIV testing. Also, they can buy HIVST kits online if needed. After completion of the 12-month follow-up, these participants can obtain a free supply of HIVST kits for a 12-month period. The same procedure offered to the intervention group will be implemented for the control group after follow-up evaluations are completed.

\section{Follow-up of positive results}

Participants who have positive results for themselves or their sexual partners using HIVST kits will be advised to contact the RAs by the 24-hour telephone hotline or WeChat. The RAs will provide additional consultation about the testing results and recommend them to have confirmatory laboratory tests in local CDC. Transfer services to the local CDC and professional psychological counselling institute will be provided, as necessary. If the participants are confirmed to be HIV positive, the process of follow-up data collection will be discontinued for them.

\section{Outcome measures}

The primary outcome is the mean number of HIV tests used by MSM from baseline to the 12-month evaluation. This number will be calculated based on the total number of HIVST kits and site-based HIV tests reported by participants. The secondary outcome is the mean number of HIV tests for sexual partners within 12 months reported by MSM, using the same calculations. The tertiary outcomes are the frequency of consistent condom usage for anal sex (never/sometimes/usually/always using condoms), 
and the numbers of reported sexual partners during the past 12 months.

\section{Data collection}

Data will be collected anonymously through online questionnaires produced by sojump (http://www. sojump.com), a professional online survey company, which has a confidential contract with the study team to encrypt the data and ensure the safety and privacy of the participants' information. An account with sojump will be set up, and the password will only be accessed by the RAs. Participants in the intervention and control groups will receive and complete the questionnaires via WeChat five times, at baseline and 3-, 6-, 9- and 12-month follow-ups.

The content validity of the baseline questionnaire was obtained by expert review (including two experts from CDC, two experts on clinical psychology and one expert on HIV/AIDS prevention and control). Included in this questionnaire are items related to social-demographic information, HIV testing history (number of HIV tests, test results and reasons for site-based HIV tests/self-tests), partner testing history (number of tests with HIVST kits distributed by MSM and site-based HIV tests recommended by MSM, test results and number of sexual partners refusing to test) and high-risk sexual behaviour history (number of sexual partners, number of and reasons for condomless oral or anal sex), during the past year and last 3 months. Two previously pilottested items assess HIV testing self-efficacy (Cronbach's $\alpha=0.74$ ) with statements about confidence in HIVST and confidence in regular HIV testing (every 6 months). Response choices are based on a 10-point Likert-type scale ranging from $1=$ not at all confident to $10=$ completely confident.

The follow-up questionnaires, to be administered at 3-month intervals, include items from the baseline measure evaluating HIV testing, partner testing, highrisk sexual behaviour during the past 3 months and self-efficacy for HIV testing. In addition, data will be collected from men in both groups about the sources of obtaining HIV self-test kits, such as from our study, online purchase or their friends. Participants who report positive or uncertain results on HIVST will be asked whether they have linked to care for confirmatory laboratory HIV test and antiretroviral treatment.

At the 12-month follow-up, participants will also be asked questions about their experiences with selftesting and their willingness to use HIVST kits in the future. Compensation (US $\$ 16$ per person) will be provided to participants for their time spent after filling out the questionnaires each time.

If any follow-up questionnaires have not been completed, we will remind participants via WeChat and/or text messages three times at intervals of 1,2 and 3 weeks after the questionnaires have been sent.

\section{Statistical analysis plan}

Analysis plan

Analyses will be conducted using SPSS V.18.0. Data will be imported directly to SPSS V.18.0 from sojump, avoiding data inputting errors. Statistical results will be reported with $p$ values and $95 \%$ CI for the corresponding hypothesis tests. $\mathrm{P}$ values $<0.05$ are considered statistically significant. Baseline characteristics of the two groups will be compared. Analyses will be performed between the intervention and control groups for recent and non-recent testers.

The primary analysis will compare the mean number of HIV tests within 12 months by using independent sample $t$-tests. At the end of the study, generalised estimated equation models will be used to analyse the variation tendency of the mean number of HIV tests every 3 months for participants. We will also use independent sample $t$-tests to analyse the mean number of HIV tests for sexual partners of MSM.

The frequency of high-risk sexual behaviours every 3 months will be analysed using a generalised estimated equation model. The relationship between the results of HIVST (positive, negative or uncertain) and condom use rate after the test will be explored using logistic regression analysis. The trend of HIV testing self-efficacy will be explored with generalised estimated equation models. The rate of positive selftesting results and the linkage to care (the rate of MSM who have confirmatory laboratory testing and initiate antiretroviral treatment in $\mathrm{CDC}$ ) will be analysed descriptively.

\section{Sample size}

The target sample size was calculated using G-power V.3. $0^{41}$ with a one-tailed test, $80 \%$ power and a $5 \%$ significance level. Calculations of sample size were made with consideration of previous research findings in China ${ }^{42}$ indicating the mean number of HIV tests for recent testers was 1.35 tests per year. Guidelines recommend individuals at high risk have 2-4 HIV tests per year, ${ }^{33} 34$ thus an increase from 1.35 to 2 tests per year (SD 1.61) ${ }^{42}$ is anticipated for recent testers. The mean number of HIV tests for non-recent testers is 0.2 tests per year based on findings of past research in Australia. ${ }^{25}$ Thus, we expect to detect an increase from 0.2 to 1 test per year (SD 0.7$)^{25}$ for non-recent testers. Given a $20 \%$ missing rate, we conservatively estimate 184 recent testers and 26 non-recent testers will help detect the difference in HIV testing frequency between the two arms.

\section{Study schedule}

Two years (14 December 2017-13 December 2019) will be needed to complete the study, including 6 months to recruit participants, 1 year for follow-up and collecting data and 6 months to process the data and report. 


\section{Patient and public involvement}

We took participants' priorities into account by conducting interviews for $23 \mathrm{MSM}$ to explore their experience and asking their preference for delivery methods. Results from interviews indicated that $89.5 \%$ (17/19) MSM who had HIV self-tests preferred fingerprick-based testing kits, and all MSM expressed belief that express mail was a great way to receive HIVST kits if no information about AIDS or HIV showed on the package. This information helped us to design our study and choose the appropriate delivery method. But these men were not involved in the study design. In addition, they were not involved in the study recruitment and conduction either. Potential participants have the right to refuse to participate in this study after assessing the burden of the intervention (including long follow-up process and time spent in completing questionnaires for five times). All participants will maintain rights to access services at the CDC or CBO. After the study is completed, the results will be disseminated to the study population.

\section{Ethics and dissemination}

The study has received an ethical approval. Moreover, the following measures will be taken to meet ethical standards: (1) oral informed consent for rapid HIV testing at the time of screening and written informed consent before enrolment will be required, and (2) personal information of participants will be stored in an offline encrypted computer that can only be accessed by the research team. Results from this study will be disseminated through conferences and academic journals.

\section{TRIAL REGISTRATION}

The study has been registered with Chinese Clinical Trial Registry (trial ID: ChiCTR1800015584, on 9 April 2018).

\section{DISCUSSION}

The HIV epidemic is rapidly growing among MSM, ${ }^{3-5}$ yet HIV testing uptake remains far below the recommended level in China and other countries. ${ }^{39}$ Due to diverse socioeconomic status, the coverage of sitebased HIV testing services differs by country and region. As a complementary approach, HIVST has the potential to expand availability of basic HIV testing services and broader HIV testing coverage. ${ }^{43}$ However, HIVST has not been widely used around the world. ${ }^{44}$ One of the main reasons is lack of enough rigorous evidence on the implementation of HIVST to promote HIV testing, which will aggravate the concerns for potential hazards of HIVST without supervision. ${ }^{42}$

This study is designed to explore the effectiveness of free HIVST kits availability on increasing HIV testing frequency among MSM in a resource-limited country, as previous RCTs have been performed only in developed countries. ${ }^{24} 25$ Several types of HIVST kits have been approved by China's regulation department. ${ }^{38}$ However, there are no relevant policies for use and regulation of the self-testing kits. China is exploring strategic approaches to integrate HIVST with sitebased HIV testing services. This RCT study targets both recent testers and non-recent testers and HIV testing frequencies for the latter group are far below the recommended level. If successful, the study will provide evidence for policymakers to scale up the HIVST approach to reach more high-risk individuals. The evidence from this study could also be used to develop tailored and contextualised HIV prevention strategies for Chinese MSM.

A major innovation of this study is to determine the effects of distribution of HIVST kits from MSM to their sexual partners on enlarging HIV testing coverage. HIV-positive MSM who were infected through regular or casual sexual partners accounted for an increasing proportion of newly infected individuals. ${ }^{23}$ Although partner testing (encouraging MSM to bring their partners to have HIV tests) has been recommended by WHO since $2012,{ }^{45}$ few data are available to assess the implementation of this policy. ${ }^{46}$ The approach of selftesting has the potential to improve partner testing for HIV due to its convenience and privacy. However, to our best knowledge, there is no study reporting the effectiveness for partner testing with distribution of free HIVST kits among MSM in China. Evidence from this study will help to guide the application of HIVST kits to promote partner testing and scale up HIV testing coverage.

The major challenge of this study is to maintain our participants for 12 months. Previous longitudinal studies reported about $18 \%$ of participants were lost to follow-up among MSM in China. ${ }^{47}$ To address this problem, we will enlarge the sample size by $20 \%$, and contact participants every 3 months by asking them to fill out the follow-up questionnaires. Moreover, data from the sequenced follow-up evaluations could demonstrate dynamic changes in HIV testing frequency, and high-risk sexual behaviour, which may expand understanding about implementation of HIVST among MSM and their sexual partners.

This study has several limitations. First, participants must have a rapid HIV test (only HIV negative individuals will be enrolled) at recruitment settings prior to entering the study, which will exclude some MSM who live far from study settings or are concerned about stigma. Second, free HIVST kits will be provided, and thus the result cannot be generalised directly to the community, where HIVST kits are not free or may be expensive. Third, finger-prick-based testing kits will be used, so the results may not apply to oral-fluid-based kits. A fourth limitation is that data about sexual partners' use of HIVST kits will be obtained indirectly by report of MSM without direct verification. A final 
limitation is that the sample of non-recent testers is small $(n=26)$ which may limit generalisability for this group.

Acknowledgements We thank the staff in Zuo An Cai Hong (the gay-friendly community-based organisation) for their assistance in recruitment for this study.

Contributors $\mathrm{CZ}$ and $\mathrm{XL}$ initiated and designed this protocol, with DK-G and LAG providing expertise on the methodology of the study. $C Z$ and $X L$ helped with the design of the questionnaires. JZ provided the support of statistical analysis. All authors edited the protocol and agreed with the final manuscript.

Funding This study was supported by Central South University Graduate Independent Exploration Innovation project (2018zzts885), Central South University Innovation-driven project (2018CX036) and Fogarty International (D43 TW009579).

Competing interests None declared.

Patient consent Obtained.

Ethics approval The Institutional Review Board of Behavioral and Nursing Research in Xiangya School of Nursing of Central South University, China (approvalnumber 2018002).

Provenance and peer review Not commissioned; externally peer reviewed.

Open access This is an open access article distributed in accordance with the Creative Commons Attribution Non Commercial (CC BY-NC 4.0) license, which permits others to distribute, remix, adapt, build upon this work non-commercially, and license their derivative works on different terms, provided the original work is properly cited, appropriate credit is given, any changes made indicated, and the use is non-commercial. See: http://creativecommons.org/licenses/by-nc/4.0/.

\section{REFERENCES}

1. World Health Organization. Global Health Observatory (GHO) data. Geneva, Switzerland: WHO, 2017

2. UNAIDS. AIDS by the numbers: AIDS is not over, but it can be. Geneva, Switzerland: UNAIDS, 2016.

3. UNAIDS. The global HIVIAIDS epidemic. Geneva, Switzerland: UNAIDS, 2017

4. The Chinese Center for Disease Control and Prevention. Important fact about HIV/AIDS. 2014 http://www.chinacdc.cn/jkzt/crb/zl/azb/ zstd/201412/t20141201_107282.html (accessed 9 Mar 2018).

5. Qin Q, Tang W, Ge L, et al. Changing trend of HIV, syphilis and hepatitis $\mathrm{C}$ among men who have sex with men in China. Sci Rep 2016;6:31081.

6. Tang S, Tang W, Meyers K, et al. HIV epidemiology and responses among men who have sex with men and transgender individuals in China: a scoping review. BMC Infect Dis 2016;16:588.

7. The Chinese Center for Disease Control and Prevention. Update on the AIDS/STDS epidemic in China in December 2017. $2017 \mathrm{http}: / /$ kns.cnki.net/KCMS/detail/11.4818.R.20180207.1451.001.html?uid= WEEvREcwSIJHSIdRa1FhcEE0RVZxejRjRktOVSsyZjZoc2Z5YXZq UW01UT0=\$9A4hF_YAuvQ5obgVAqNKPCYcEjKensW4ggl8Fm4gTk oUKaID8j8gFw!!v=MDY2MDJuTXJZOUNaT3NQWXc5TXptUm42ajU3 VDNmbHFXTTBDTEw3UjdxZWIrWnNGeXpsVTc3TEIGND1QUy9T YjdHNEg5 (accessed 9 Mar 2018).

8. UNAIDS. Fast-track: ending the AIDS epidemic by 2030. Geneva Switzerland: UNAIDS, 2014.

9. Zou $\mathrm{H}, \mathrm{Hu} \mathrm{N}$, Xin Q, et al. HIV testing among men who have sex with men in China: a systematic review and meta-analysis. AIDS Behav 2012;16:1717-28.

10. Zou H, Wu Z, Yu J, et al. Sexual risk behaviors and HIV infection among men who have sex with men who use the internet in Beijing and Urumqi, China. J Acquir Immune Defic Syndr 2010;53(Suppl 1):S81-7.

11. Wang HY, Xu JJ, Zou HC, et al. Sexual risk behaviors and hiv infection among men who have sex with men and women in China: evidence from a systematic review and meta-analysis. Biomed Res Int 2015;2015:1-12.

12. Valdiserri RO. Late HIV diagnosis: bad medicine and worse public health. PLoS Med 2007;4:e200.

13. World Health Organization. Guidelines on HIV self-testing and partner notification: supplement to consolidated guidelines on HIV testing services. Geneva, Switzerland: WHO, 2016.

14. Pant Pai N, Sharma J, Shivkumar S, et al. Supervised and unsupervised self-testing for HIV in high- and low-risk populations: a systematic review. PLoS Med 2013;10:e1001414.
15. Frye V, Wilton L, Hirshfied S, et al. "Just because it's out there, people aren't going to use it." HIV self-testing among young, black msm, and transgender women. AIDS Patient Care STDS 2015;29:617-24.

16. Bilardi JE, Walker S, Read T, et al. Gay and bisexual men's views on rapid self-testing for HIV. AIDS Behav 2013;17:2093-9.

17. UNAIDS. The gap report [EB/OL]. Geneva, Switzerland: UNAIDS, 2015.

18. Figueroa $\mathrm{C}$, Johnson $\mathrm{C}$, Verster $\mathrm{A}$, et al. Attitudes and acceptability on hiv self-testing among key populations: a literature review. AIDS Behav 2015;19:1949-65.

19. Greacen T, Friboulet D, Fugon L, et al. Access to and use of unauthorised online HIV self-tests by internet-using Frenchspeaking men who have sex with men. Sex Transm Infect 2012;88:368-74.

20. Wong HT, Tam HY, Chan DP, et al. Usage and acceptability of HIV self-testing in men who have sex with men in Hong Kong. AIDS Behav 2015;19:505-15.

21. Huang E, Marlin RW, Young SD, et al. Using grindr, a smartphone social-networking application, to increase HIV self-testing among black and Latino men who have sex with men in Los Angeles, 2014. AIDS Educ Prev 2016;28:341-50.

22. Wei $\mathrm{C}$, Yan $\mathrm{H}$, Yang $\mathrm{C}$, et al. Accessing HIV testing and treatment among men who have sex with men in China: a qualitative study. AIDS Care 2014;26:372-8.

23. UNAIDS. UNAIDS Data. Geneva, Switzerland: UNAIDS, 20172017.

24. Katz D, Golden M, Hughes J, et al. HIV self-testing increases HIV testing frequency among high-risk men who have sex with men: a randomized controlled trial [abstract]. Vancouver, Canada: 8th International AIDS Society Conference, 2015.

25. Jamil MS, Prestage G, Fairley CK, et al. Effect of availability of HIV self-testing on HIV testing frequency in gay and bisexual men at high risk of infection (FORTH): a waiting-list randomised controlled trial. Lancet HIV 2017;4:e241-50.

26. Sharma A, Chavez PR, MacGowan RJ, et al. Willingness to distribute free rapid home HIV test kits and to test with social or sexual network associates among men who have sex with men in the United States. AIDS Care 2017;29:1499-503.

27. Lf L. HIV incidence and using of oral rapid test kits for regular male sexual partner among men who have sex with men in Beijing. Hebei Medical University, 2017 http://kns.cnki.net/KCMS/detail/ detail.aspx?dbcode=CMFD\&dbname=CMFD201801\&filename $=$ 1017826881.nh\&uid=WEEvREcwSIJHSIdRa1FhdXNXaOhHRXZiaHBo WVJieWNUcEdpajJJMVAwMD0=\$9A4hF_YAuvQ5obgVAqNKPC YcEjKensW4ggl8Fm4gTkoUKalD8j8gFw!!\&v=MzAyODhaT1pwRkNq bFZMek1WRjI2R2J1NkdObkVycEViUEISOGVYMUx1eFITNORoMVQz cVRyV00xRnJDVVJMS2Y= (accessed 9 Mar 2018).

28. Thirumurthy $\mathrm{H}$, Masters $\mathrm{SH}$, Mavedzenge $\mathrm{SN}$, et al. Promoting male partner HIV testing and safer sexual decision making through secondary distribution of self-tests by HIV-negative female sex workers and women receiving antenatal and post-partum care in Kenya: a cohort study. Lancet HIV 2016;3:e266-74.

29. Ministry of Health of the People's Republic of China. 2016 China AIDS response progress report. 2016 http://hunan.sina.com.cn/ news/2016-11-27/detail-ifxyawmp0324958.shtml?from=hunan_ydph (accessed 9 Mar 2018).

30. HIV epidemic in Hunan Province at the end of October 2016 (in Chinese). http://www.eol.cn/hunan/hunannews/201611/t20161128_ 1471024.shtml (accessed 9 March 2018).

31. Wang XD, Jiang $\mathrm{CQ}$, Ma H. Rating scales for mental health. Beijing, MA: Chinese journal of mental health, 1999:263-6.

32. Huang ZL, Shan F, Yan RL. Analysis of HIV/AIDS high risk behavior and influencing factors among men who have sex with men in Changsha. Practical Preventive Med 2013;20:1435-8.

33. Patel RR, Patel S, Clarke E, et al. Guidance and practice on frequency of HIV and sexually transmitted infection testing in men who have sex with men - what is the European situation? Int J STD AIDS 2014;25:213-8.

34. Templeton DJ, Read P, Varma R, et al. Australian sexually transmissible infection and HIV testing guidelines for asymptomatic men who have sex with men 2014: a review of the evidence. Sex Health 2014;11:217-29.

35. Choko AT, Desmond N, Webb EL, et al. The uptake and accuracy of oral kits for HIV self-testing in high HIV prevalence setting: a cross-sectional feasibility study in Blantyre, Malawi. PLoS Med 2011;8:e1001102.

36. Li Y, Wang Y, Zhang R, et al. [Analysis on accuracy and influencing factors of oral fluid-based rapid HIV self-testing among men who have sex with men]. Zhonghua Liu Xing Bing Xue Za Zhi 2016;37:72-5.

37. Hurt CB, Powers KA. Self-testing for HIV and its impact on public health. Sex Transm Dis 2014;41:10-12. 
38. Yan L, Xiao PP, Yan HJ, et al. [The advance of detection technology of HIV self-testing]. Zhonghua Yu Fang Yi Xue Za Zhi 2017;51:1053-6.

39. Alere. Alere determine HIV-1/2 quick reference card-global (English). https://www.alere.com/zh/zh/product-details/determine-hiv-1-2.html (accessed 9 Mar 2018).

40. Faul F, Erdfelder E, Lang AG, et al. G*Power 3: a flexible statistical power analysis program for the social, behavioral, and biomedical sciences. Behav Res Methods 2007;39:175-91.

41. Zhong F, Tang W, Cheng W, et al. Acceptability and feasibility of a social entrepreneurship testing model to promote HIV self-testing and linkage to care among men who have sex with men. HIV Med 2017;18:376-82.

42. Alere. Alere determine HIV-1/2 brochure-global (English). https:// www.alere.com/zh/zh/product-details/determine-hiv-1-2.html (accessed 9 Mar 2018).

43. Zhang C, Li X, Brecht ML, et al. Can self-testing increase HIV testing among men who have sex with men: A systematic review and metaanalysis. PLoS One 2017;12:e0188890.
44. Campbell S, Klein R. Home testing to detect human immunodeficiency virus: boon or bane? J Clin Microbiol 2006;44:3473-6.

45. World Health Organization. Guidance on couples HIV testing and counselling - including antiretroviral therapy for treatment and prevention in serodiscordant couples: recommendations for a public health approach. Geneva, Switzerland: WHO, 2012.

46. World Health Organization. Global health sector response to HIV, 2000-2015: focus on innovations in Africa: progress report. Geneva, Switzerland: WHO, 2015.

47. Yan HJ, Yang HT, Zhao JK, et al. Long-chain peer referral of men who have sex with men: a novel approach to establish and maintain a cohort to measure HIV incidence, Nanjing, China. J Acquir Immune Defic Syndr 2012;2:177-84.

48. Chen J, Li X, Xiong Y, et al. Reducing the risk of HIV transmission among men who have sex with men: a feasibility study of the motivational interviewing counseling method. Nurs Health $\mathrm{Sc}$ 2016;18:400-7. 